(Aus dem physikalischen Institut des wissenschaftlichen Instituts zu Moskau.)

\title{
Untersuchungen über die Ionentheorie der Reizung.
}

\author{
II. Mitteilung.
}

\section{Die Ionentheorie der Reizung und die Pflügerschen Gesetze.}

$$
\text { Von }
$$

Dr. P. Lasareff.

Mitglied der Akademie der Wissemschaften zu Petrograd, Professor an der Universität unal Technischen Hochschule zi Muskat.

Mit 1 Textabbildungr.

(Eingegungen an 1. September 19:1.)

In meinen früheren Arbeiten habe ich cine Theorie der Reizungen aufgestellt, die cine Weiterentwicklung der Theorie von Prof. Nernst und Prof. Loeb ist, und habe bewiesen, daß dir Formel für die Gesctzo der minimalen Reizungen folgende ist:

$$
C_{1}^{C_{1}}=K^{\prime}
$$

wobei ('i die Konzentration dere erregenden Ionen ist. ('2 die Konzentration der erregungshemmenden Ionen, und $K^{\prime}$ cine Konstante; die Formel muß als Grundgesetz für dic Roizung der lebenden Gewebr gelten $\left.{ }^{1}\right)$.

Djeses Gesetz (das Loebsche Gesetz) bericht sich auf die Reizschwelle und begründet folglich die Beziehungen zwischen den $C_{1}^{-}$und den $C_{2}$ - Iomenkonzentrationen, die erforderlich sind, um eine minimale Reizung zu erzielen.

Der Wert $K^{\prime}$ ist verschieden für die verschiedenen Gewebe und kann durch experimentelle Untersuchungen genau bestimmt werden.

Das durch die Formel (1) ausgedrückte und theoretisch in meinen Arbeiten 1910 für alle Rcizungen abgeleitete Gesetz ist schon früher ron Loe b 1906 enpurisch für den Einfluß von Salzlösungen auf Muskeln und Nerven gefunden worden, und ich erlaube mir diese's Gesetz das Loebsche Gesetz zu nonnen.

Indem ich cliese Formel (1) auf die Reizung der Muskeln und Nerver durch den elektrischen Strom anwanite, habe ich gefunden, dab alle Nernstschen Geretze sich aus dem Loebschen Gesetz ableiten lassen. Auch habe ich diesses Gesetz als Basis für die Theorie des Dunkelsehens

1) P. Lasareff, Pflügers Arch. f. d. ges. Physiol. 135, 196. 1910. 
genommen ${ }^{1}$ ), und die vollständige Übereinstimmung zwischen Theorie und Praxis erlaubt uns diese Gleichung als eine Grundformel für Reizungen anzusehen. Schließlich ist dieses Grundgesetz der Reizungen von Loeb durch sehr exakte und feine Untersuchungen der Bewegungen des Balanus iberneus geprüft worden ${ }^{2}$ ), und hat sich innerhalb der Grenzen der möglichen Beobachtungsfehler als genau erfüllt erwiesen. Man kann also dieses Loebsche Gesetz als das Grundgesetz der Reizungen für lebendes Gewebe ansehen.

Dieser Umstand erlaubt uns anzunehmen, daß, wenn in normalem ungereiztem Zustande die Gleichung $\frac{C_{1}}{C_{2}}=K_{0}$ ist, und, um einen Reiz hervorzurufen, $\begin{aligned} & C_{1} \\ & C_{2}\end{aligned}=K^{\prime}>K_{0}$ sein muß, bei vergrößertem $\frac{C_{1}}{C_{2}}$ auch die Reizbarkeit verstärkt wird, eine Verkleinerung des Wertes $K^{\prime}$ dagegen auch eine verringerte Reizbarkeit nach sich zieht. Diese Betrachtungen dienen uns als Basis zum Beweis des Pflügerschen Gesetzes.

Die animalischen Gewebe enthalten eine gewisse Anzahl von Ionen, die beim Durchleiten des elektrischen Stromes ihre Stellung verändern, bei geschlossenem konstanten Strome also überholen die beweglicheren Ionen die weniger beweglichen, so daß das Verhältnis der Ionenkonzentrationen, welches im Ruhestand bestand, sich an den Elektroden verändern muß. Den Untersuchungen von Loeb zufolge sind es die K-Ionen, die die Gewebe reizen, und ihre Tätigkcit wird durch die Ca- (oder Mg-) Ionen herabgesetzt. Da die K-Ionen beweglicher sind als die Ca-Ionen, so vergrößert sich das Verhältnis $\frac{C_{1}}{C_{2}}$ an der Kathode, wohin sich die $\mathrm{K}$ - und die Ca-Ionen hinbewegen.

An der Kathode muß sich die Reizbarkeit vergrößern, an der Anode findet das Gegenteil statt; die Ca-Ionen bleiben hier in größcren Mengen zurück als die K-Ionen, also muß sich hier die Reizbarkeit verringern. Wenn der polarisierende Strom soweit verstärkt wird, daß der Reiz eintritt, so ist aus dem vorhergehenden leicht zu ersehen, daß sich der Reiz bei Schließung des Stromes zuerst an der Kathode bemerkbar machen muß. Bei Unterbrechung des Stromes diffundieren die an der Kathode angesammelten Ionen der Metalle zur Anode, und dann stellen sich hier, infolge der größeren Diffussionsgeschwindigkeit der K-Ionen, die für den minimalen Reiz erforderlichen Verbältnisse der reizenden und erregungshemmenden Ionen ein, daraus geht hervor, da $\beta$ bei Unterbrechung des Stromes sich der Reiz an der Anode einstellen muß.

Wenn der Strom konstant geworden ist, können wir Reizveränderungen auch in quantitativer Form erhalten, wenn wir annehmen, daß der elektrische Strom durch das Protoplasma (Neuroplasma) and die

1) P. Lasareff, Pflügers Arch. f. d. ges. Physiol. 15\&, 459. 1913.

2) S. Loeb, Proc. of the nat. acad. of sciences (U.S. A) 1, 439. 1915. 
darin enthaltenen Fibrillen geleitet ist. Die Leitungsfähigkeit dieser beiden Teile der Nervenfaser ist nicht gleich, und wir haben allen Grund anzunehmen, daß die Leitungsfähigkeit der Fibrillen die größere ist; die Stromlinien müssen also so verteilt sein wie es Abb. 1 zeigt.

Der mittlere Teil $F F$ stellt schematisch die Fibrille dar, die sie umgebenden Teile $N N-N N$ das Protoplasma. Die Stromlinien gehen von einer Elektrode $P_{1}$ zur anderen $P_{2}$.

An den Punkten $A$ und $B$, wo die Stromlinien in die Fibrille ein- und wieder austreten, müssen

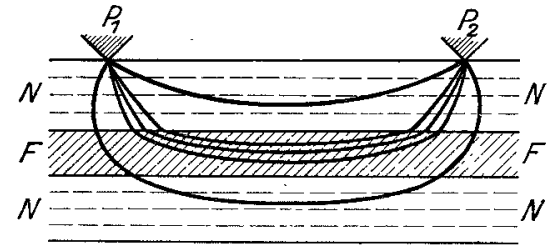

Abh. 1. sich die Ionen ansammeln. da die Fibrillenmembran für sie undurchlässig ist; infolgedessen muß sich auch die Verteilung der Ionen in dem Raume $A B$ unter dem Finflusse eines konstanten stromes ändern. Wir können uns also den Nerv in der Form eines Zylinders vorstellen, an dessen Grundflächen Elektroden angebracht sind; weil die Fibrille wehr dünn ist. ist die Ansammlung der lonen an den halbdurchlïssigen Membranen auf dem ganzen Durebmesser des Nervs fast gleichmäßig. und wir können die obenerwähnten 'infache'n Bedingungen als zutreffend betrachten. In diesem Fall mub überall für cine jede der beiden Ionenarten, die sich in einer sehr whwarhen Lösung finden, die Diffusionsgleichung

$$
d t=k \frac{c^{2} c}{i x^{2}}
$$

senügen, wobei die Richtang $x$ von dem Berührungwpunkte der einen Elektrode der Fibrille entlang zur anderen als poritiv angesehen wird: $k$ ist der Diffusionskoeffizient und $t$ die Zcit. Bci stationärem Zustance ist $\frac{\partial C}{\partial t}=0$. Wir müssen also auf der ganzen streneke des reizharen Genchess

$$
\begin{aligned}
& \partial^{2} C \\
& i x^{2}
\end{aligned}=0 \quad \text { haben. }
$$

Das Integral dieser Gleichung hat für allo Ionenarten die Form

$$
C=A x+B
$$

Dieses Integral muß den Bedingungen genügen, die $x=0$ e entsprechen. Bei stationärem Zustancle des Stromleiters muß die zur Elektrode durch den Strom angeführte Salzquantität, die den Betrag $i v$ hat $(i=$ die Intensität des Ntromes, $v=$ ('ine Konstante), dem Diffusionsstrom, der das Salz fortführt und ressen Ausdruck $k \frac{\partial Q}{\partial x}$ ist, gleich rein, so dab.

$$
k \frac{\partial C}{c x}=y^{\prime} i
$$


ist. Indem wir $\frac{\partial C}{\partial x}$ bestimmen (Gleichung 2) und in die Gleichung (3) einsetzen, finden wir

$$
A=\frac{\nu}{k} i
$$

Um den Wert von $B$ zu finden, schreiben wir, daß die Ionenzahl zwischen den Elektroden vor und nach dem Durchgang des Stromes unveränderlich ist und nehmen an, daß die Ionen von außen nicht in den Raum, zwischen den Elektroden eindringen können.

Die Zahl der Ionen vor Durchgang des Stromes ist gleich dem Produkt der Konzentration $C_{0}$ mit dem Volumen des Raumes zwischen den Elektroden; dieses letztere ist gleich dem Produkt des Abstandes der Elektroden $a$ mit dem Querschnitt der Fibrille $\sigma$, folglich ist die Zahl der Ionen vor Durchgang des Stromes $C_{0} \sigma a$ gleich.

Um den entsprechenden Wert nach Durchgang des Stromes zu bestimmen müssen wir den Wert $C \sigma \cdot d x$, welcher die Ionenzahl in einem Zylinder, dessen Grundfläche gleich $\sigma$ und dessen Höhe $d x$ ist, von 0 bis $a$ integrieren; auf diese Weise ist die Zahl der Ionen im Zy. linder gleich

$$
\int_{0}^{a} C \sigma d x=\sigma \int_{0}^{a}[A x+B] d x=\frac{\sigma \nu i a^{2}}{2 k}+\sigma a B .
$$

Da dieser Wert, welcher die Anzahl der Ionen nach dem Durchgang des Stromes darstellt, unverändert bleibt, so ist

$$
C_{0}=\frac{v i a}{2 k}+B \quad \text { oder } \quad B=C_{0}-\frac{v a}{2 k} i .
$$

Indem wir die Werte von $B(5)$ und von $A(4)$ in die Gleichung (2) einsetzen, finden wir für $C$ den Wert

$$
C=C_{0}+\left[x-\begin{array}{l}
a \\
2
\end{array}\right] \stackrel{v i}{k}
$$

Wenn die Ionenkonzentrationen schwach sind, so muß die Diffusion der einen und der anderen Ionen als voneinander unabhängig betrachtet werden; folglich muß die Gleichung (6) sowohl für die reizhervorrufenden Ionen $C_{1}$ als auch für die reizhemmenden Ionen $C_{2}$ erfüllt sein, so daß

$$
C_{1}=C_{0}^{\prime}+\frac{v_{1} i}{k_{1}}\left[x-\frac{a}{2}\right]
$$

und

$$
C_{2}=C_{0}^{\prime \prime}+\frac{p_{9} i}{k_{2}}\left[x-\frac{a}{2}\right]
$$

ist, wobei $C_{0}^{\prime}$ und $C_{0}^{\prime \prime}$ die Konzentrationen der einen und der anderen Ionen in dem nichtgereizten Nerv darstellen. 
Das Verhältnis $\frac{C_{1}}{C_{2}}$, welches die Reizbarkeit bestimmt, ist gleich

$$
K^{\prime}=\frac{C_{1}}{C_{2}}=\frac{C_{0}^{\prime}\left\{1+\frac{\nu_{1} i}{k_{1} C_{0}^{\prime}}\left[x-\frac{a}{2}\right]\right\}}{C_{0}^{\prime \prime}\left\{1+\frac{\nu_{2} i}{k_{2} C_{0}^{\prime \prime}}\left[x-\frac{a}{2}\right]\right\}} .
$$

Es ist leicht zu sehen, daß, wenn $x=\frac{a}{2}$ ist, das Verhältnis ${\stackrel{C}{C_{2}}}_{C_{2}}$ sich in $C_{0}^{\prime \prime}$ verwandelt, mit anderen Worten, daß der Reizbarkeitszustand dem normalen Reizbarlieitszustande entspricht.

Die Lage des neuen Punktes entspricht also theoretisch immer der Mitte des Abstandes zwischen den Elektroden, praktisch aber verschiebt sich derselbe mit der Verstärkung des Stromes gegen die Kathode hin. Eine Erklärung dieser Erscheinung muß darin gesucht werden, dal erstens die Diffusion der Ionen bei den Konzentrationen, welche in dem Leiter bestehen. nicht voneinander unabhängig sind, und clals zweitens stärkere Ströme die Anzahl der Ionen zwischen den Elektroden ändern können. Was die Änderung der Reizbarkeit vom neutralen Punkt zur Anode und Kathode hin betrifft, so finden wir, indem wir diesen Punkt als Nullpunkt für ein neues Koordinatensystem annehmen, also $x \cdots c_{2} \quad-x_{1}$ setzen, dali

ist.

$$
C_{2}^{\prime}=\frac{\left.C_{0}^{\prime} \mid 1+\frac{\nu_{1}^{\prime}}{k_{1} C_{0}^{\prime}} \cdot i x_{1}\right]}{C_{0}^{\prime \prime}\left[1+\frac{\nu_{2}}{k_{2} C_{0}^{\prime \prime}} i x_{1}\right]}
$$

Führen wir die Division aus, so finden wir

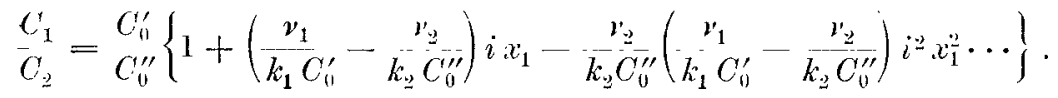

Wenn $x_{1}$ nicht groß ist und $\begin{gathered}\nu_{1} \\ k_{1} C_{11}^{\prime} \cdots\end{gathered} k_{2} C_{0}^{\prime \prime}$ positiv ist, was wir für den Muskel und den Nerv annehmen müssen, so zeigt die vorstehende Gleichung, daß das Verhältnis $\frac{C_{1}}{C_{2}}$ und folglich auch die Reizbarkeit von dem neutralen Punkte aus gegen die Kathode hin wächst und zur Anode hin abnimmt. 